\section{P72 UNDERSTANDING WHY UK GPS ARE LEAVING GENERAL PRACTICE-A SYSTEMATIC REVIEW OF QUALITATIVE RESEARCH}

${ }^{1} \mathrm{~L}$ Long*, ${ }^{2} \mathrm{~A}$ Sansom, ${ }^{3} \mathrm{~A}$ Aylward, ${ }^{1} \mathrm{~S}$ Robinson, ${ }^{1} \mathrm{R}$ Anderson, ${ }^{2} \mathrm{E}$ Fletcher, ${ }^{4} \mathrm{~J}$ Welsman, ${ }^{5} \mathrm{~S}$ Dean, ${ }^{2} \mathrm{~J}$ Campbell. 'Evidence Synthesis and Modelling for Health Improvement (ESMI), University of Exeter Medical School, Exeter, UK; ${ }^{2}$ Primary Care Research Group, University of Exeter Medical School, Exeter, UK; ${ }^{3}$ Patient and Public Involvement representative, University of Exeter Medical School, Exeter, UK; ${ }^{4}$ Centre for Biomedical Modelling and Analysis, University of Exeter, Exeter, UK; ${ }^{5}$ Psychology Applied to Health (PAtH), University of Exeter Medical School, Exeter, UK

\subsection{6/jech-2017-SSMAbstracts. 173}

Background UK GPs are quitting direct patient care. The research presented here is part of a wider mixed methods study (ReGROUP) which focuses on retention of the experienced GP workforce, and on supporting the return to work of GPs following a career break. We undertook a qualitative synthesis of the evidence to identify factors that affect GPs' retention in the workforce and that affect GPs' decisions to quit direct patient care, take career breaks from general practice, and return to general practice after a career break

Methods Five UK interview-based studies were identified and quality assessed using the adapted "Wallace tool". A thematic synthesis was performed using NVivo software and a detailed explanatory model was constructed to provide an overview of the key contexts and factors affecting UK GPs in practice. Applicability of the explanatory model was verified by patient representatives in a patient and public involvement (PPI) workshop as well as by stakeholders from the wider research team.

Results Three central dynamics key to understanding UK GP quitting behaviour emerged - factors associated with low job satisfaction, high job satisfaction, and those linked to the doctor-patient relationship. The importance of contextual influence, especially the changing nature of clinical practice, was noted. GPs with high job satisfaction describe feeling supported by good practice relationships, while GPs with poor job satisfaction report feeling overworked and unsupported.

Combined with changing relationships with patients and interfaces with secondary care, and the gradual sense of loss of control over large parts of the job, many GPs report a reduction in job satisfaction. Once job satisfaction has become negatively impacted, the combined pressures of increased patient demand and workload together with other stress factors has left many feeling unsupported and vulnerable to burnout and ill health, and, ultimately, to the decision to leave general practice.

Conclusion Job satisfaction appears to be a key indicator of whether a GP will successfully adapt and remain in practice, or will become overwhelmed by external influences and pressures and leave the profession. Many GPs report that job satisfaction directly relates to the quality of the doctor-patient relationship, and is dependent on the time available for GPs to spend with their patients.

\section{P73 AVOIDABLE EMERGENCY ADMISSIONS IN THE REPUBLIC OF IRELAND: ANALYSIS OF REGIONAL DETERMINANTS}

B Lynch*, AP Fitzgerald, JP Browne. Epidemiology and Public Health, UCC, Cork, Ireland

\subsection{6/jech-2017-SSMAbstracts.174}

Background Many emergency admissions are deemed to be avoidable. There is a potential for several conditions, including angina, diabetes and chronic obstructive pulmonary disease (COPD), to be more appropriately treated in community and urgent care settings. The rate of emergency admissions for these conditions can be influenced by a number of factors. These include factors directly linked to health services, such as access to general practice, ambulance resources, emergency departments and hospitals. Other factors beyond the control of health services, such as deprivation and rurality, may also play a role.

Methods Directly age-standardised emergency admission rates were constructed at a county level for a series of urgent conditions for 2013-2015.

Based on methods adopted by a UK study, we examined the role of service level factors on these rates (block 2: availability and accessibility of services) having removed the effect of non-modifiable by service factors (block 1: population, geography and health factors).

A multivariate regression using forward stepwise regression within each block was used, beginning with variables from block 1. We then examined the association between the residuals from the first model and block 2 service level variables. Factors were included in the final model if the $p$-value was $<0.05$.

Results There were 228435 emergency admissions for selected urgent conditions in 2013-2015. They accounted for $24.5 \%$ of all emergency admissions. Results from the first stepwise regression identified one significant non-modifiable by services factor, the regional unemployment rate. This accounted for $30 \%$ of the observed variation $\left(\mathrm{R}^{2}=30 \%\right)$. Preliminary analysis indicates that factors concerning availability and access to primary care services and hospitals accounted for an additional $47 \%$ of the remaining variation (total $\left.\mathrm{R}^{2}=63 \%\right)$. The factors identified as significant were: the percentage of total emergency admissions to hospital with a length of stay of exactly 1 day; the number of general practice nurses per 100000 population; and, the percentage of general practitioners that had effective access to social workers.

Conclusion Having allowed for non-modifiable by service factors, almost half of the variation in emergency admissions for urgent conditions was explained by the availability of primary care and hospital resources. Therefore, increased access to, and use of, community-based primary care may reduce the number of inappropriate admissions. 